\title{
Outcomes of Acute Gastrointestinal Bleeding in Patients With COVID-19: A Case-Control Study
}

\author{
Umair Iqbal $^{\mathrm{a},}$ e, Pooja D. Patel ${ }^{\mathrm{b}}$, Christopher A. Pluskota ${ }^{\mathrm{c}}$, Andrea L. Berger ${ }^{\mathrm{d}}$, \\ Harshit S. Khara ${ }^{\mathrm{a}}$, Bradley D. Confer ${ }^{\mathrm{a}}$
}

\begin{abstract}
Background: Coronavirus disease 2019 (COVID-19) patients are at higher risk of acute gastrointestinal bleeding (AGIB) due to higher use of steroids, mechanical ventilation, and use of anticoagulation. We performed this study to compare outcomes of AGIB in COVID19-positive patients and those without COVID-19 and AGIB.

Methods: This was a case-control study including patients admitted from March 2020 to February 2021 with the diagnosis of AGIB. Patients were divided into two groups: COVID-19-positive and nonCOVID-19 patients. Our primary outcomes were in-hospital or 30 days mortality and length of stay. Secondary outcomes were the rate of rebleeding, the need for intensive care unit (ICU) level of care, and the need for blood transfusion.
\end{abstract}

Results: Eighteen COVID-19-positive patients and 54 matched nonCOVID-19 patients were included. The COVID-19-positive patients less frequently had endoscopies performed $(33.3 \%$ vs. $74.1 \%, \mathrm{P}=$ $0.0059)$ and had greater steroid use $(83.3 \%$ vs. $14.8 \%, \mathrm{P}<0.0001)$ compared to non-COVID-19 patients. ICU stays were more likely in the COVID-positive patients (odds ratio (OR): 20.41; 95\% confidence interval $(\mathrm{CI}): 2.59-160.69 ; \mathrm{P}=0.004)$ as was longer hospital length of stay (OR: 1.08; 95\% CI: $1.03-1.13$; P = 0.002). Mortality, readmission within 30 days, need for blood transfusion, and having rebleeding during the admission did not differ for COVID-19 and non-COVID-19 patients.

Conclusion: COVID-19 patients with AGIB are more likely to require ICU admission and had a longer length of stay. Despite the sig-

Manuscript submitted November 13, 2021, accepted December 21, 2021

Published online January 10, 2022

aDepartment of Gastroenterology and Hepatology, Geisinger Medical Center, Danville, PA, USA

bDepartment of Internal Medicine, Geisinger Medical Center, Danville, PA, USA

'Department of Gastroenterology and Hepatology, University hospitals, Cleveland, OH, USA

${ }^{\mathrm{d}}$ Department of Population Health Sciences, Geisinger Medical Center, Danville, PA, USA

${ }^{e}$ Corresponding Author: Umair Iqbal, Department of Gastroenterology and Hepatology, Geisinger Medical Center, Danville, PA 17821, USA.

Email: uiqbal@geisinger.edu

doi: https://doi.org/10.14740/gr1483 nificantly lower rate of endoscopic procedures performed in patients with COVID-19, need for blood transfusion, mortality and rebleeding were not significantly different.

Keywords: COVID-19; Acute gastrointestinal bleeding; Mortality; Upper GI bleeding; Lower GI bleeding

\section{Introduction}

Coronavirus disease 2019 (COVID-19) has affected more than 50 million patients in the United States of America and is associated with significant morbidity and mortality. COVID-19 patients are at relatively higher risk of acute gastrointestinal bleeding (AGIB) than the general population $[1,2]$. This is likely secondary to higher use of corticosteroids which is effective in the management of COVID-19, need for mechanical ventilation which increases the risk of stress ulcers and use of anticoagulation for the treatment and prevention of thrombotic complications of the virus [3-5]. A recent observational study including 1,206 COVID-19 patients with AGIB showed these patients are at high risk of AGIB with an overall incidence of $3.1 \%$ [6]. However, AGIB was not found to be the independent predictor of mortality in COVID-19 patients. In another study including 11,158 hospitalized COVID-19 patients, prevalence of AGIB was 3\%. The study showed higher mortality in hospitalized COVID-19 patients with AGIB [7].

Guidelines recommend endoscopic evaluation within 24 $\mathrm{h}$ of presentation of GI bleeding [8,9]. However, conservative management alone without endoscopic intervention has been shown to be successful in the management of COVID-19 patients [10-12]. However, no prior study to our knowledge directly compared outcomes of COVID-19 patients with GI bleeding with non-COVID 19 patients with GI bleeding. The purpose of our study was to describe the characteristics and outcomes of COVID-19-positive patients with AGIB compared to matched controls with acute GI bleeding without COVID-19.

\section{Materials and Methods}

This was a single-center case-control study done at a tertiary care hospital in rural Pennsylvania. Patients who were admit- 
ted to the hospital with the diagnosis of AGIB between March 2020 and February 2021 were included. Patients were divided into two groups: COVID-19-positive and non-COVID-19 patients. All patients over 18 years old and who had signs and symptoms of AGIB during the admission were included in the study. Patients in the COVID-19 group had a COVID-19 reverse transcription-polymerase chain reaction (RT-PCR) positive at the time of admission or 14 days within the diagnosis of AGIB. Patients under 18 years of age or without any evidence of AGIB were excluded from the study. Eighteen COVID19-positive patients (cases) and 133 non-COVID-19 patients (controls) met the initial study criteria. We then performed matching without replacement by age ( \pm 10 years) and gender. The study was designed for a 1:3 case/control ratio. Five controls were matched to each case. Strata identification numbers were assigned to each cluster of cases and their matches. The study team performed a manual chart review on the first three controls that met inclusion criteria. Extra controls were included in the matching in the event that a non-COVID-19 patient did not meet inclusion criteria upon chart review. The final analysis dataset had 18 COVID-19-positive patients and 54 non-COVID-19 patients. Our primary outcomes were mortality and need for intensive care unit (ICU) stay. Secondary outcomes were length of stay, risk of rebleeding, need for vasopressors and 30-day readmission rates.

Patient characteristics and outcomes are summarized using means and standard deviations or medians and interquartile ranges (IQRs) for continuous variables and frequency counts and percentages for categorical variables. Conditional logistic regression models were used to compare the characteristics and outcomes of cases and controls and took into account matched cluster groups with a statement that identified the strata identification numbers. The association between outcomes and COVID-19 status was estimated using conditional logistic regression models and odds ratios (ORs) and $95 \%$ confidence intervals (CIs) are reported. Five patients with insufficient follow-up time to assess rebleeding were excluded from the analysis of that outcome. Subgroup analysis was done to compare COVID-19-positive patients with and without endoscopy done. Student's $t$-tests and Wilcoxon rank sum tests were used to compare continuous variables and Pearson's Chisquare or Fisher's exact tests were used to compare categorical variables. SAS 9.4 (Cary, NC) was used for analysis. P-values $<0.05$ were considered statistically significant.

The study was approved by Institutional Review Board, and was conducted in compliance with the ethical standards of the responsible institution on human subjects as well as with the Helsinki Declaration.

\section{Results}

Eighteen COVID-19-positive patients and 54 matched nonCOVID-19-positive patients were included in the analysis. For the entire cohort, the mean age was 69.7 years and $66.7 \%$ were male. COVID-19 patients had greater steroid use $(83.3 \%$ vs. $14.8 \%, \mathrm{P}<0.0001$ ) compared to non-COVID-19 patients. There was no significant difference in the use of anticoagulation, non-steroidal anti-inflammatory drugs (NSAIDs), and proton pump inhibitor (PPI) use between the two groups. There was no difference in hemoglobin $(8.9$ vs. $8.6 ; \mathrm{P}=0.76)$ and international normalized ratio (INR) (1.4 vs. $1.3 ; \mathrm{P}=0.1286)$ on presentation between the two groups. Melena was the most common presentation and was present in $52.8 \%$ of patients followed by hematochezia in $38.9 \%$ and coffee ground emesis in $9.7 \%$ of patients. Upper GI bleeding was suspected in $62.5 \%$ of the patient and lower GI bleeding in $37.5 \%$ of the patients. There was no difference in Glasgow Blatchford Score in patients with suspected upper GI bleeding on presentation between the two groups. There was no difference in comorbidities including hypertension, diabetes, and cirrhosis between the two groups. Baseline characteristics and outcomes are shown in Table 1.

There were a total of 46 patients who underwent endoscopic evaluation. There was no difference in the need for endoscopic intervention to achieve hemostasis between the two groups. The COVID-19-positive patients less frequently had endoscopies performed (33.3\% vs. $74.1 \%, \mathrm{P}=0.0059)$. The most common endoscopic finding in both groups was peptic ulcer disease which was found in 12 patients. Other endoscopic finding included diverticulosis in seven patients, gastritis in four patients, small bowel arteriovenous malformation (AVMs) in two patients which required argon plasma coagulation (APC), esophageal varices in two patients which require endoscopic band ligation, gastroesophageal junction mass in two patients which was biopsied and Mallory Weiss tear, Cameron ulceration, dieulafoy lesion, ischemic small bowel ulcers, post polypectomy bleeding, solitary ulcer at hepatic flexure and sigmoid ulceration in one patient each. ICU stays were more likely in COVID-19-positive patients (OR: 20.41; 95\% CI: 2.59 - 160.69) as was longer hospital length of stay (OR: 1.08; 95\% CI: 1.03 - 1.13). Mortality trended higher in the COVID-19 group but did not reach statistical significance $(38.9 \%$ vs. $18.5 \%$; $\mathrm{P}=0.0549)$. Only one death was directly attributed to GI bleeding in COVID-19 group compared to two patients who died secondary to GI bleeding in non-COVID-19 group. Vasopressor requirements was also higher in the COVID-19 group but did not reach statistical significance $(44.4 \%$ vs. $25.9 \% ; \mathrm{P}=0.14)$. There was no statistically significant difference in 30 days readmission rates, blood transfusion requirements, and risk of rebleeding for COVID-19 and nonCOVID-19 patients. Table 2 summarizes the difference in outcomes between the two groups.

In a subgroup analysis among the 18 COVID-19 patients, six $(33.3 \%)$ underwent endoscopy and 12 (66.7\%) were managed conservatively. Mortality, readmission, rebleeding, ICU stay, need for vasopressors, and hospital length of stay were not significantly different between the groups. There was also no statistically significant difference in ICU admission and mortality with the use of anticoagulation in COVID-19 patients.

\section{Discussion}

Our study revealed that patients with COVID-19 have a higher length of stay and risk of ICU admission. This is because COVID-19 patients are at higher risk of respiratory failure and 
Table 1. Baseline Characteristics of COVID-19 and Non-COVID-19 Patients on Presentation

\begin{tabular}{|c|c|c|c|c|}
\hline & $\begin{array}{l}\text { Total GI bleed } \\
\text { patients }(\mathrm{N}=72)\end{array}$ & COVID-19 $(\mathrm{N}=18)$ & $\begin{array}{l}\text { Non-COVID-19 } \\
\text { controls }(\mathrm{N}=54)\end{array}$ & P-value \\
\hline Age at admission, mean (SD) & $69.7(11.74)$ & $68.8(13.31)$ & $70.0(11.29)$ & m.v. \\
\hline Sex, n (\%) & & & & m.v. \\
\hline Male, n (\%) & $48(66.7 \%)$ & $12(66.7 \%)$ & $36(66.7 \%)$ & m.v. \\
\hline Hemoglobin at presentation, mean (SD) & $8.7(2.87)$ & $8.9(2.82)$ & $8.6(2.91)$ & 0.7639 \\
\hline Anticoagulation, n (\%) & & & & 0.3549 \\
\hline Prophylactic dose & $14(19.4 \%)$ & $5(27.8 \%)$ & $9(16.7 \%)$ & \\
\hline Therapeutic dose & $28(38.9 \%)$ & $8(44.4 \%)$ & $20(37.0 \%)$ & \\
\hline None & $30(41.7 \%)$ & $5(27.8 \%)$ & $25(46.3 \%)$ & \\
\hline Melena, n (\%) & $38(52.8 \%)$ & $11(61.1 \%)$ & $27(50.0 \%)$ & 0.4408 \\
\hline Coffee ground emesis, $\mathrm{n}(\%)$ & $7(9.7 \%)$ & $3(16.7 \%)$ & $4(7.4 \%)$ & 0.2439 \\
\hline $\begin{array}{l}\text { Hematochezia, melena, or coffee } \\
\text { grounds emesis, } n(\%)\end{array}$ & $65(90.3 \%)$ & $18(100.0 \%)$ & $47(87.0 \%)$ & 0.1523 \\
\hline NSAIDs, n (\%) & $38(52.8 \%)$ & $10(55.6 \%)$ & $28(51.9 \%)$ & 0.7774 \\
\hline Hypertension, n (\%) & $48(66.7 \%)$ & $13(72.2 \%)$ & $35(64.8 \%)$ & 0.5186 \\
\hline Cirrhosis, n (\%) & $11(15.3 \%)$ & $1(5.6 \%)$ & $10(18.5 \%)$ & 0.2083 \\
\hline Diabetes, n (\%) & $25(34.7 \%)$ & $5(27.8 \%)$ & $20(37.0 \%)$ & 0.4776 \\
\hline History of GI bleed, n (\%) & $24(33.3 \%)$ & $4(22.2 \%)$ & $20(37.0 \%)$ & 0.2275 \\
\hline
\end{tabular}

*Use of steroids was significantly higher in COVID-19 patients. COVID-19: coronavirus disease 2019; GI: gastrointestinal; SD: standard deviation; INR: international normalized ratio; IQR: interquartile range; m.v.: matching variable; PPI: proton pump inhibitor; NSAIDs: non-steroidal anti-inflammatory drugs.

therefore more likely to require admission to critical care unit and have a higher length of stays compared to non-COVID-19 patients. There was no statistically significant difference in mortality between COVID-19 and non-COVID-19 patients with AGIB in our study. However, it was trended higher in COVID-19 patients (38.9\% vs. $18.5 \%)$. This may be due to the small sample size of the study and larger studies may show COVID-19 patients with AGIB are at higher risk of mortality compared to non-COVID-19 patients. However, higher mortality in COVID-19 patients is likely secondary to respiratory failure and critical illness as seen in prior studies and may not be directly secondary to AGIB [13]. Our study also revealed that patients with COVID-19 are less likely to undergo endoscopic evaluation for GI bleeding. In our experience, this is due to two major reasons. First, in the pre-vaccination era, there was a high amount of anxiety among healthcare professionals in the management of these patients due to the high risk of transmission, high mortality, and lack of personal protective equipment early in the pandemic $[14,15]$. Secondly, early data on COVID-19 patients with GI bleeding showed promising results with conservative management [10-12, 16, 17]. In a study including 24 COVID-19 patients with GI bleeding, conserva- tive management alone without any endoscopic intervention was successful in controlling bleeding in all the patients [10]. Similar results were in another study including 11 patients with lower GI bleeding, and conservative management was successful in the majority of these patients [16]. Therefore, there was a strong inclination towards conservative management and limiting endoscopic evaluation for hemodynamically stable COVID-19 patients. In a recently published systematic review and meta-analysis, we revealed that $59 \%$ of the COVID-19 patients with AGIB were managed conservatively and less than one-third (31.5\%) underwent endoscopic evaluation. Although pooled overall mortality was high $19.1 \%$, pooled mortality directly secondary to AGIB was $3.5 \%$ and pooled risk of rebleeding was $11.3 \%$ [17].

Conservative management is also reasonable as the majority of these patients have respiratory failure or at high risk for decompensation and unnecessary or non-urgent endoscopic evaluation may worsen their respiratory status and increase the risk of mechanical ventilation. Our study results are in agreement with prior published studies that revealed conservative management alone with endoscopic intervention may be sufficient in these patients as mortality, blood transfu- 
Table 2. Comparative Outcomes Between COVID-19 Patients With Non-COVID-19 Patients

\begin{tabular}{|c|c|c|c|c|}
\hline & $\begin{array}{l}\text { Total GI bleed } \\
\text { patients }(\mathrm{N}=72)\end{array}$ & COVID-19 $(\mathrm{N}=18)$ & $\begin{array}{l}\text { Non-COVID-19 } \\
\text { controls }(\mathrm{N}=54)\end{array}$ & P-value \\
\hline Endoscopy done, n (\%) & $46(63.9 \%)$ & $6(33.3 \%)$ & $40(74.1 \%)$ & 0.0059 \\
\hline Intervention, $\mathrm{n}(\%)$ & $18(25.0 \%)$ & $\begin{array}{l}4(22.2 \%) \\
\text { PUD }=2 \text { (one patient required } \\
\text { hemospray and other required } \\
\text { epinephrine injection and } \\
\text { clips for hemostasis). Small } \\
\text { bowel AVM in one patient } \\
\text { which required APC. Solitary } \\
\text { ulcer at hepatic flexure } \\
\text { which required OTSC. }\end{array}$ & $\begin{array}{l}14(25.9 \%) \\
\text { APC for small bowel AVMs } \\
\text { in one patient, biopsy of GE } \\
\text { junction mass. Hemostatic clips, } \\
\text { bipolar coagulation, epinephrine } \\
\text { injection and hemospray for } \\
\text { PUD, variceal banding in two } \\
\text { patients, sigmoid colon biopsy } \\
\text { for suspected ischemia. }\end{array}$ & 0.7683 \\
\hline $\begin{array}{l}\text { Repeat endoscopy (among patients } \\
\text { with initial endoscopy), n (\%) }\end{array}$ & $14(30.4 \%)$ & $2(33.3 \%)$ & $12(30.0 \%)$ & 0.8145 \\
\hline Inpatient or 30-day mortality, n (\%) & $17(23.6 \%)$ & $7(38.9 \%)$ & $10(18.5 \%)$ & \\
\hline Causes of mortality & & $\begin{array}{l}\text { Septic shock, sepsis, COVID-19 } \\
\text { pneumonia, acute hypoxic } \\
\text { respiratory failure, AGIB }\end{array}$ & $\begin{array}{l}\text { AGIB, hemorrhagic shock, } \\
\text { septic shock, ischemic bowel, } \\
\text { multiorgan failure, CVA, AML, } \\
\text { metastatic renal cell carcinoma }\end{array}$ & \\
\hline $\begin{array}{l}\text { Rebleeding during admission } \\
\text { among patients with sufficient } \\
\text { observation time, } \mathrm{n}(\%)\end{array}$ & $8(11.9 \%)$ & $2(14.3 \%)$ & $6(11.3 \%)$ & 0.6837 \\
\hline Pressors, n (\%) & $22(30.6 \%)$ & $8(44.4 \%)$ & $14(25.9 \%)$ & 0.1404 \\
\hline Length of stay (days), median (IQR) & $7.0(4.0-18.5)$ & $21.5(7.0-39.0)$ & $6.0(4.0-13.0)$ & \\
\hline
\end{tabular}

*There was no statistically significant difference in mortality between COVID-19 patients and non-COVID-19 patient. ICU admission and length of stay were higher in COVID-19 patients. COVID-19: coronavirus disease 2019; GI: gastrointestinal; IQR: interquartile range; AVM: arteriovenous malformations; APC: argon plasma coagulation; OTSC: over the scope clip; ICU: intensive care unit; PUD: peptic ulcer disease; GE: gastroesophageal; CVA: cerebrovascular accident; AML: acute myeloid leukemia; GBS: Glasgow Blatchford Score; AGIB: acute gastrointestinal bleeding.

sion requirements, rebleeding, and need for vasopressors were not significant differences between the two groups. Although need for critical care stay and length of stay were significantly higher in COVID-19 groups, this was not directly secondary to GI bleeding but rather is multifactorial as COVID-19 patients are more likely to require mechanical ventilation and have complicated hospital course compared to non-COVID-19 patients.

As per our literature review, this is the first study that directly compared the outcomes of COVID-19 patients with GI bleeding with non-COVID-19 patients. Our study provides clinicians with differences in outcomes including mortality in COVID-19 patients with non-COVID-19 patients, common etiologies of AGIB in COVID-19 patients, and the importance of conservative management in COVID-19 patients with AGIB. Our study will help guide clinicians in their decision-making regarding the management of AGIB in COV-
ID-19 patients including the timing of endoscopic evaluation in these patients. Our study has some limitations including the retrospective design of the study which may have introduced bias in the study results. Secondly, the sample size of the COVID-19 group is small. As we did not include patients with no AGIB in our study, we could not compare outcomes of COVID-19 patients with AGIB with COVID-19 patients without AGIB to evaluate if AGIB is associated with higher mortality in COVID-19 patients. Therefore, further studies are needed to evaluate if AGIB in COVID-19 patients is associated with higher mortality. Though our study period included two major peaks of the COVID-19 pandemic in the United States of America, the majority of these were admitted before the advent of the COVID-19 vaccination. This might have impacted the clinical approach of the gastroenterologist. It will be interesting to see the comparative outcomes in the post-vaccination era and if they had impacted the outcomes 
of GI bleeding. Our study results will also be helpful in some developing countries with limited resources which currently have a huge burden of COVID-19 with a limited vaccinated population to make their clinical decision in managing $\mathrm{COV}$ ID-19 patients.

In conclusion, COVID-19 patients with AGIB are more likely to require ICU admission and had a longer length of stay. Despite the significantly lower rate of endoscopic procedures performed in COVID-19 patients, need for blood transfusion, mortality, and rebleeding were not significantly different, and conservative management alone may be sufficient in these patients. However, clinical judgment should be utilized in each case and endoscopic evaluation should be considered in hemodynamically unstable patients and patients at high risk of hemodynamic instability.

\section{Acknowledgments}

None to declare.

\section{Financial Disclosure}

None to declare.

\section{Conflict of Interest}

None to declare.

\section{Informed Consent}

Not applicable.

\section{Author Contributions}

Umair Iqbal was involved in writing the full manuscript. Pooja Patel was involved in data collection process. Christopher Pluskota was involved in data collection process. Andrea L. Berger did the statistical analysis of the paper. Harshit S. Khara and Bradley D. Confer are the senior authors and were involved in supervising the study and finalizing the manuscript. All authors agreed to the final version of the manuscript.

\section{Data Availability}

The authors declare that data supporting the findings of this study are available within the article.

\section{References}

1. Martin TA, Wan DW, Hajifathalian K, Tewani S, Shah SL, Mehta A, Kaplan A, et al. Gastrointestinal bleeding in pa- tients with coronavirus disease 2019: a matched case-control study. Am J Gastroenterol. 2020;115(10):1609-1616.

2. Lin L, Jiang X, Zhang Z, Huang S, Zhang Z, Fang Z, $\mathrm{Gu} Z$, et al. Gastrointestinal symptoms of 95 cases with SARS-CoV-2 infection. Gut. 2020;69(6):997-1001.

3. RECOVERY Collaborative Group, Horby P, Lim WS, Emberson JR, Mafham M, Bell JL, Linsell L, et al. Dexamethasone in hospitalized patients with COVID-19. N Engl J Med. 2021;384(8):693-704.

4. Richardson S, Hirsch JS, Narasimhan M, Crawford JM, McGinn T, Davidson KW, the Northwell C-RC, et al. Presenting characteristics, comorbidities, and outcomes among 5700 patients hospitalized with COVID-19 in the New York City area. JAMA. 2020;323(20):2052-2059.

5. Jenner WJ, Kanji R, Mirsadraee S, Gue YX, Price S, Prasad S, Gorog DA. Thrombotic complications in 2928 patients with COVID-19 treated in intensive care: a systematic review. J Thromb Thrombolysis. 2021;51(3):595607.

6. Makker J, Mantri N, Patel HK, Abbas H, Baiomi A, Sun $\mathrm{H}$, Choi Y, et al. The incidence and mortality impact of gastrointestinal bleeding in hospitalized COVID-19 patients. Clin Exp Gastroenterol. 2021;14:405-411.

7. Trindade AJ, Izard S, Coppa K, Hirsch JS, Lee C, Satapathy SK, Northwell C-RC. Gastrointestinal bleeding in hospitalized COVID-19 patients: a propensity score matched cohort study. J Intern Med. 2021;289(6):887894.

8. Strate LL, Gralnek IM. ACG clinical guideline: management of patients with acute lower gastrointestinal bleeding. Am J Gastroenterol. 2016;111(5):755.

9. Laine L, Barkun AN, Saltzman JR, Martel M, Leontiadis GI. ACG Clinical Guideline: Upper Gastrointestinal and Ulcer Bleeding. Am J Gastroenterol. 2021;116(5):899917.

10. Shalimar, Vaishnav M, Elhence A, Kumar R, Mohta S, Palle C, Kumar P, et al. Outcome of conservative therapy in coronavirus disease-2019 patients presenting with gastrointestinal bleeding. J Clin Exp Hepatol. 2021; 11(3):327-333.

11. Melazzini F, Lenti MV, Mauro A, De Grazia F, Di Sabatino A. Peptic ulcer disease as a common cause of bleeding in patients with coronavirus disease 2019. Am J Gastroenterol. 2020;115(7):1139-1140.

12. Barrett LF, Lo KB, Stanek SR, Walter JW. Self-limited gastrointestinal bleeding in COVID-19. Clin Res Hepatol Gastroenterol. 2020;44(4):e77-e80.

13. Sharma S, Badami V, Rojas E, Mittal A, Stansbury R, Rana B, Wilson A, et al. ICU mortality in patients with coronavirus disease 2019 infection: highlighting healthcare disparities in rural Appalachia. Crit Care Explor. 2021;3(10): e547.

14. Mosheva M, Hertz-Palmor N, Dorman Ilan S, Matalon N, Pessach IM, Afek A, Ziv A, et al. Anxiety, pandemicrelated stress and resilience among physicians during the COVID-19 pandemic. Depress Anxiety. 2020;37(10):965971.

15. Shanafelt T, Ripp J, Trockel M. Understanding and addressing sources of anxiety among health care profes- 
sionals during the COVID-19 Pandemic. JAMA. 2020; 323(21):2133-2134.

16. Holzwanger EA, Bilal M, Stallwood CG, Sterling MJ, Yacavone RF. Acute lower gastrointestinal bleeding during the COVID-19 pandemic - less is more! Endoscopy.
2020;52(9):816-817.

17. Iqbal U, Anwar H, Siddiqui HU, Khan MA, Kamal F, Confer BD, Khara HS. Acute gastrointestinal bleeding in COVID-19 patients: a systematic review and meta-analysis. Clin Endosc. 2021;54(4):534-541. 